\title{
EXTENSIONS OF GROUP REPRESENTATIONS OVER FIELDS OF PRIME CHARACTERISTIC
}

\author{
BURTON FEIN ${ }^{1}$
}

Let $G$ be a finite group having a normal Hall subgroup $H$, let $K$ be a field, and let $T$ be an irreducible (linear) $K$-representation of $H$ of degree $\operatorname{deg} T$ whose character is invariant under the action of $G$. We say that $T$ is extendible to $G$ if there exists a $K$-representation $S$ of $G$ such that $S(h)=T(h)$ for all $h \in H$. In [5, Theorem 6] Gallagher proved that $T$ is extendible if $K$ is the field of complex numbers. The case when $K$ is an arbitrary field of characteristic zero is treated by Isaacs in [7]. In this note we show that the arguments in Isaacs' paper can be extended to yield the following result:

Theorem. If $K$ has characteristic $p, p>0$, and if either $H$ is $p$-solvable or $(\operatorname{deg} T,[G: H])=1$, then $T$ is extendible to $G$.

The following lemma is essentially a corollary of the Swan-Fong theorem [8, Theorem 6]. An elementary proof of this result appears in [2].

Lemma 1. Let $D$ denote the set of all finite groups $B$ with the property that if $H$ is a subgroup of $B$, then the degrees of the absolutely irreducible linear and absolutely irreducible projective $K$-representations of $H$ divide the order $|H|$ of $H$. If $G$ is a finite group such that every composition factor of $G$ lies in $D$, then the degrees of the absolutely irreducible linear $K$-representations of $G$ divide $|G|$. In particular, if $K$ has characteristic $p$ and $G$ is p-solvable, the degrees of the absolutely irreducible linear $K$-representations of $G$ divide $|G|$.

Proof. The first part of the lemma is easily proved by induction using the argument of Theorem 53.17 of [1]. The statement about $p$-solvable groups now follows from [1, Theorems 27.28 and 53.3] together with the remarks in $[1$, p. 600].

LеммA 2. Let $W$ be an absolutely irreducible representation of $H$ which is a constituent of $T$. If $(\operatorname{deg} T,[G: H])=1$, then $(\operatorname{deg} W,[G: H])=1$.

Received by the editors January 13, 1969 and, in revised form, March 5, 1969.

1 The preparation of this paper was supported in part by NSF Grant GP-8622. The author is grateful to Dr. Isaacs for making available a copy of his paper [7] prior to its publication and to Dr. Isaacs and Dr. G. J. Janusz for several helpful suggestions. 
Proof. This follows immediately from the theory of the Schur index [3, Theorem 1.4].

If $V$ is a representation of a group $B$ we denote by det $V$ the onedimensional representation of $B$ obtained by taking the determinant of $V$. det $V$ is an element of the group of one-dimensional representations of $B$ and we denote by $o(V)$ the order of det $V$ in this group.

Lemma 3. Let $K$ be algebraically closed of characteristic $p$ and suppose that $(\operatorname{deg} T,[G: H])=1$. Then there is a unique extension $S$ of $T$ to $G$ such that $(o(S),[G: H])=1$.

Proof. The fact that $T$ is extendible to $G$ follows from the proof of Satz 17.12 on p. 572 in [6]. Let $U$ be some extension of $T$ to $G$. The proof of Theorem 51.7 of [1] shows that if $S$ is any extension of $T$ to $G$ then there exists a projective representation $Y$ of $G / H$ such that $S(g) \times U(g) \times Y(g)$ (Kronecker product) for all $g \in G$. Thus $Y$ is a one-dimensional linear representation of $G / H$. The result now follows from the proof of [5, Theorem 5].

Proof of the THeorem. Let $E=K(\sqrt[n]{1}), n=|G|$. Let $W$ be an irreducible $E$-representation of $H$ which is a constituent of $T$ and let $\theta$ be the character of $W$. From the theory of the Schur index we have

$$
T^{E} \sim \sum_{\oplus} W^{\sigma}
$$

the sum ranging over all automorphisms $\sigma$ in the Galois group $\mathcal{G}(K(\theta) \mid K)$ of $K(\theta)$ over $K[3$, Theorem 1.4]. Since $K(\theta) \subset E$, $\mathcal{G}(K(\theta) \mid K)$ is cyclic. Thus $I$, the inertia group of $\theta$, is a normal subgroup of $G, H \subset I \subset G$ and $G / I$ is cyclic [7, Lemma 1.2]. By Lemmas 1,2 , and 3 there is a unique extension $\hat{W}$ of $W$ to $I$ such that $(o(\hat{W}),[I: H])=1$. Let $V=\hat{W}^{G} . V$ is an irreducible $E$-representation of $G$. Let $\chi$ be the character of $V$. Then $V$ is realizable in $K(\chi)$. If $\tau \in \mathcal{G}(K(\chi) \mid K), \tau \neq 1$, then $\left.V\right|_{H}$ and $\left.V^{\tau}\right|_{H}$ have no constituents in common [7, Proposition 1.4]. Let $S$ be an irreducible $K$-representation of $G$ such that $S^{E} \sim \sum_{\oplus} V^{\tau}$ thesum ranging over all $\tau \in \mathcal{G}(K(\chi) \mid K)$. It suffices to prove that $\left.S\right|_{H} \sim T$. $T$ is a constituent of $\left.S\right|_{H}$, so by Clifford's Theorem $\left.S\right|_{H} \sim e T$.

The remarks above show that $e$ is also the multiplicity with which $W$ occurs as a constituent of $\left.V\right|_{H}$. The multiplicity of $\hat{W}$ in $\left.V\right|_{I}$ is 1 , $\hat{W}$ is the unique constituent of $\left.V\right|_{I}$ whose restriction to $H$ is $W$. Thus $e=1$, which proves the result.

REMARK. Lemma 3 has an application to the question of determining when an indecomposable $K$-representation of $H$ is extendible to a $K$-representation of $G$. Assume that $H$ is $p$-solvable and $K$ is alge- 
braically closed of characteristic $p,(p,[G: H])=1$. Let $U$ be a principal indecomposable $K[H]$-module such that $U^{(\sigma)} \cong U$ for all $g \in G$. Let $M$ be the unique minimal submodule of $U$. Clearly $M^{(0)} \cong M$ for all $g \in G$ so by Lemma $3, M$ is extendible to a $K[G]$-module $N, N_{H}$ $=M$. Let $V$ be a principal indecomposable $K[G]$-module having $N$ as its unique minimal submodule. In view of Theorem (2B) of [4], to prove that $V_{H}=U$ it is sufficient to show that $U \mid V_{H}$. Let $R$ be the sum of the irreducible $K[H]$-submodules of $V_{H}$. Then $M=N_{H} \subset R$ so $M$ is a direct summand of $R$. But $V_{H}$ is injective and so contains an injective hull of $M[1$, Theorem 57.13]. Since $U$ is the injective hull of $M$ we have proved that $U$ is extendible to $V$.

\section{REFERENCES}

1. C. W. Curtis and I. Reiner, Representation theory of finite groups and associative algebras, Interscience, New York, 1962.

2. E. C. Dade, Degrees of modular irreducible representations of p-solvable groups, Math. Z. 104 (1968), 141-143.

3. B. Fein, Representations of direct products of finite groups, Pacific J. Math. 20 (1967), 45-58.

4. P. Fong, Solvable groups and modular representation theory, Trans. Amer. Math. Soc. 103 (1962), 484-494.

5. P. X. Gallagher, Group characters and normal Hall subgroups, Nagoya Math. J. 20 (1962), 223-230.

6. B. Huppert, Endliche Gruppen. I, Springer-Verlag, Berlin, 1967.

7. I. M. Isaacs, Extensions of group representations over nonalgebraically closed fields, Trans. Amer. Math. Soc. 141 (1969), 211-228.

8. R. Swan, The Grothendieck ring of a finite group, Topology 2 (1963), 85-110.

University of California, Los Angeles 